\title{
Time dependence of AGN pair echo, and halo emission as a probe of weak extragalactic magnetic fields
}

\author{
Foteini Oikonomou* \\ Department of Physics, The Pennsylvania State University, University Park, PA 16802,USA. \\ Center for Gravitational and Particle Astrophysics, The Pennsylvania State University, \\ University Park, PA 16802,USA. \\ E-mail: oikonomouepsu.edu

\section{Kohta Murase} \\ Department of Physics, The Pennsylvania State University, University Park, PA 16802,USA. \\ Department of Astronomy \& Astrophysics, The Pennsylvania State University, University Park, \\ PA 16802,USA. \\ Center for Gravitational and Particle Astrophysics, The Pennsylvania State University, \\ University Park, PA 16802,USA. \\ E-mail: murase@psu.edu
}

\section{Kumiko Kotera}

Institut d'Astrophysique de Paris UMR7095 - CNRS, Université Pierre Marie Curie, 98 bis boulevard Arago F-75014 Paris, France.

E-mail: kotera@iap.fr

\begin{abstract}
Gamma-rays with energy exceeding $100 \mathrm{GeV}$ emitted by extragalactic sources initiate cascades in the intergalactic medium. The angular and temporal distribution of the cascade photons that arrive at the Earth depend on the strength and configuration of extragalactic magnetic fields in the line of sight. For weak enough fields, extended emission around the source (halo) is expected to be detectable, and the characteristics (angular size, energy dependence, and shape) of this emission are a sensitive probe of EGMF strength and correlation length. We model the expected spectra and angular profiles of blazars in a range of parameter space of the extra-galactic magnetic field strength and correlation length, which is unconstrained by existing measurements.
\end{abstract}

35th International Cosmic Ray Conference - ICRC2017

10-20 July, 2017

Bexco, Busan, Korea

${ }^{*}$ Speaker. 


\section{Introduction}

Magnetic fields permeate the Universe; their effects are observed in our own Galaxy, in other nearby and distant galaxies, in filaments, sheets, and clusters of large scale structure (e.g., [1, 2, $3,4]$ ), whereas there are observational hints and theoretical arguments that even the voids of large scale structure support mild magnetic fields (see e.g., [5, 6, 7] for reviews).

Despite a growing number of measurements, the configuration, coherence length (i.e. the distance over which the field direction can be considered constant) and strength of intergalactic magnetic fields (IGMFs), remains largely unknown. This hinders the efforts to understand the origin of these fields, which is largely speaking thought to either be "astrophysical", meaning generated in galaxies and astrophysical sources, or "cosmological", meaning seed fields of primordial origin. Measurements of (or limits on) the Faraday rotation of the radio emission of distant quasars, and distortions of the spectrum and polarisation of the cosmic microwave background, place an upper limit to the intergalactic magnetic field strength of the order of $\sim 10^{-9} \mathrm{G} \mathrm{Mpc}^{1 / 2}[8,9,10,11]$.

Blazars that emit $\mathrm{TeV}$ gamma-rays may provide a powerful tool for constraining the strength of IGMFs. Primary TeV gamma-rays interact with extragalactic background photons, producing high energy $e^{ \pm}$pairs. The electrons (and positrons) cool promptly through inverse Compton emission, up-scattering ambient, low-energy photons to $\sim \mathrm{GeV}$ energies. The two processes drive an electromagnetic cascade in the intergalactic medium. The cascade deposits energy to the GeV part of the spectrum, below the pair-production threshold. In the absence of intervening IGMFs, the flux expected in the $\mathrm{GeV}$ region of the blazar spectrum, is the sum of the intrinsic flux of the source in that energy range, plus the contribution from the electromagnetic cascade. However, if IGMFs are present, the electrons in the cascade are deflected, depleting the cascade signal in the GeV band. Further, if IGMFs are sufficiently weak, the secondary cascade photons, will arrive from the direction of the source, with some angular spread ("pair-halos"), and time delay ("pair-echoes").

The absence of the expected $\mathrm{GeV}$ flux in the spectra of a number of $\mathrm{TeV}$ detected blazars, has led to lower limits on the strength of intervening IGMFs, $B_{\mathrm{IGMF}} \lambda^{1 / 2} \lesssim 10^{-15} \mathrm{G} \mathrm{Mpc}^{1 / 2}$, assuming the blazar emission is steady (e.g., $[12,13,14,15])$. More conservative constraints $10^{-18}-10^{-20} \mathrm{G} \mathrm{Mpc}^{1 / 2}$ have been derived, considering that the blazar emission might be transient $[16,17,18,19,20])$.

In addition to the possible $\mathrm{GeV}$ cascade component, the deflections experienced by the cascade electrons cause an angular broadening of the arriving gamma-ray flux. If IGMFs are relatively strong, this "magnetically broadened emission" will cause the cascade flux to be significantly spread and too weak to be detectable. However, for a range of IGMFs magnetic broadening is expected to lead to a "halo" around what would be a point source in the absence of IGMFs. This halo emission, has been shown to be detectable for a range of IGMF strengths [21, 22, 23]. A measurement of the expected halo component would lead to the determination of the average IGMF strength in the line of sight.

Here we present an analytical framework to model blazar pair halo and echo emission complementary to Monte Carlo simulations, but much less computationally intensive. Where possible, we provide exact analytical expressions to calculate the cascade gamma-ray emission. We benchmark, and complement our analytical results with the results of Monte Carlo simulations, performed with the publicly available ELMAG [24]. 


\section{Methods}

Our methodology builds on the formalism developed in $[18,19,25]$. We consider a gamma-ray emitting blazar at distance $L$. Primary gamma-rays emitted at an angle $\theta_{s}$ from the jet axis, travel a distance $L^{\prime}$ before producing an $e^{ \pm}$pair, after interacting with a photon from the extragalactic background light (EBL). The electrons are deflected by an angle $\theta_{d}$ before inverse Compton scattering ambient $\mathrm{CMB}$ photons to $\mathrm{TeV}$ energies. The secondary gamma-rays will arrive at an angle $\theta_{c}$ in this picture. The geometry considered is illustrated in figure 1 . Our formalism can be applied to sources at all viewing angles $\theta_{v}$, not necessarily with a jet pointing towards the observer.

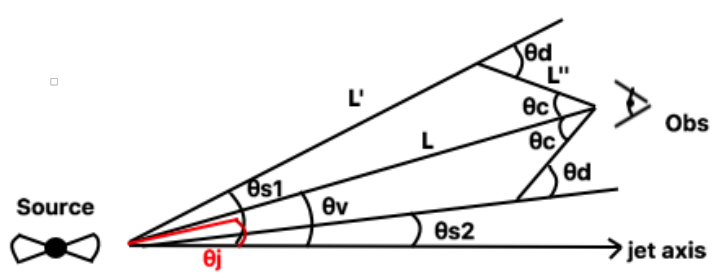

Figure 1: Geometry considered in this work, to calculate the secondary gamma-ray emission from a source at distance $L$ with a jet at viewing angle $\theta_{v}$.

The differential flux of secondary photons produced by an electron that changes Lorentz factor from $\gamma_{e}+\mathrm{d} \gamma_{\mathrm{e}}$ to $\gamma_{e}$ is given by [26]

$$
\frac{\mathrm{d} N}{\mathrm{~d} E \mathrm{~d} t}=\int \mathrm{d} \gamma_{e} \frac{\mathrm{d} N}{\mathrm{~d} \gamma_{e}} \int \mathrm{d} \varepsilon \frac{\mathrm{d} n}{\mathrm{~d} \varepsilon}\left\langle\frac{\mathrm{d} \sigma_{\mathrm{IC}}}{\mathrm{d} E} c(1-\mu)\right\rangle .
$$

Here, $E$ is the energy of the secondary photons produced by the inverse Compton process, and $\varepsilon$ the energy of the cosmic background photons. The factor $c(1-\mu)$ gives the relative velocity of the photon, and the electron, in the direction of the latter's motion, and the Klein-Nishina cross-section is given by

$$
\left\langle\frac{\mathrm{d} \sigma_{\mathrm{IC}}}{\mathrm{d} E} c(1-\mu)\right\rangle=\frac{3}{4} \sigma_{T} c \frac{1}{\gamma_{e}^{2} \varepsilon} \times\left[1+v-2 v^{2}+\frac{v^{2} w^{2}(1-v)}{2(1+v w)}+2 v \ln v\right],
$$

with $v=E /\left(4 \varepsilon \gamma_{e}^{2}(1-\xi)\right), w=4 \varepsilon \gamma_{e} /\left(m_{e} c^{2}\right)$, and $\xi=E /\left(\gamma_{e} m_{e} c^{2}\right)$, where $m_{e}$ is the electron mass, and $c$ the speed of light. We model the differential density of background photons as the sum of the CMB black body spectrum, and the cosmic infrared background (CIB) given by $\mathrm{d} n(z) / \mathrm{d} \varepsilon=$ $8 \pi \varepsilon^{2} /(h c)^{3}\left(\exp ^{\varepsilon / k T}-1\right)+\mathrm{d} n_{\mathrm{CIB}}(z) / \mathrm{d} \varepsilon$, where $T(z)$ is the CMB temperature at redshift $z, T(z)=$ $T(z=0) \cdot(1+z)$, with $T(z=0) \approx 2.73 \mathrm{~K}$, and $k$ the Boltzmann constant.

The electron energy loss rate is evaluated following

$$
\frac{1}{\gamma_{e}} \frac{\mathrm{d} \gamma_{e}}{\mathrm{~d} t}=c \int \mathrm{d} \Omega(1-\mu) \int \mathrm{d} \varepsilon \frac{\mathrm{d} n}{\mathrm{~d} \varepsilon \mathrm{d} \Omega} \kappa_{\mathrm{IC}} \sigma_{\mathrm{IC}}
$$

where $\kappa_{\mathrm{IC}}$ is the electron inelasticity in the IC process, and $\sigma_{\mathrm{IC}}$ the IC cross-section which are evaluated using the Klein-Nishina formula (eq. 2.2), $\kappa_{\mathrm{IC}} \sigma_{\mathrm{IC}}=\int \mathrm{d} E^{\prime}\left(\mathrm{d} \sigma_{\mathrm{IC}} / \mathrm{d} E^{\prime}\right) E^{\prime} /\left(\gamma_{e} m_{e} c^{2}\right)$, where $E^{\prime}$ is the primary photon energy. 
Substituting the above expressions into equation 2.1 and accounting for the intergalactic propagation of the primary gamma-rays, we get the differential flux of observed secondary photons at Earth

$$
\begin{aligned}
& \frac{\mathrm{d} N}{\mathrm{~d} E \mathrm{~d} \theta_{c}}= \int_{\theta_{f}} \int_{\gamma_{e}, \min }^{E_{\max }^{\prime} /\left(2 m_{e} c^{2}\right)} \int_{E^{\prime}=2 \gamma_{e} m_{e} c^{2}}^{E_{\max }^{\prime}} \int_{L^{\prime}=0}^{L_{\max }^{\prime}} \\
& \cdot\left[\frac{8 \pi \varepsilon^{2}}{h^{3} c^{3}} \frac{1}{e^{\varepsilon / k T(1+z)}-1}+\frac{\mathrm{d} n_{\mathrm{CIB}}(z)}{\mathrm{d} \varepsilon}\right] \\
& \cdot \mathrm{d} \varepsilon \cdot \sigma_{I C}\left(\varepsilon, E, \gamma_{e}\right) \cdot \frac{\mathrm{d} \gamma_{e}}{\mathrm{~d} t} \cdot \sin \theta_{f} \cdot \mathrm{d} \theta_{f} \cdot \mathrm{d} \varepsilon \cdot \mathrm{d} L^{\prime} \\
& \cdot \frac{e^{-L^{\prime} / \lambda\left(E^{\prime}\right)}}{\lambda\left(E^{\prime}\right)} \cdot f\left(\varepsilon, \theta_{s}\right) \cdot \frac{1}{\mathrm{~d} \theta_{c}} \cdot \exp \left(-\sqrt{L^{2}+L^{\prime 2}-2 L L^{\prime} \cos \left(\theta_{d}-\theta_{c}-\theta_{v}\right) / \lambda}(E) .\right.
\end{aligned}
$$

Here the first integral averages over pitch angles $\theta_{f}$, for which we assume a behaviour $g\left(\theta_{f}\right)=$ $\sin \left(\theta_{f}\right)$. We take the integral of $\theta_{f}$ from 0 to $\pi / 2$. The second integral runs over electron Lorentz factor $\gamma_{e}$. The upper limit on the $\gamma_{e, \max }=E_{\max }^{\prime} /\left(2 m_{e} c^{2}\right)$, where $E_{\max }^{\prime}$ is the maximum injected photon energy at the source. The third integral runs over $E^{\prime}$, the primary photon energy, with $E_{\min }^{\prime}=$ $2 \gamma_{e} m_{e} c^{2}$, assuming that the primary photon splits its energy equally between an electron and a positron. The fourth of the nested integrals runs over distance increments $\mathrm{d} L^{\prime}$, with $L_{\text {max }}^{\prime}$ depending on the source geometry (but generally $L_{\max }^{\prime}>L$ ). We calculate $\theta_{d}$, which is the deflection angle of the electrons following equation 4 of [19].

The distance at which the primary photon interacts is denoted $L^{\prime}$, with $\lambda$ the mean free path for pair production. We approximate $\lambda\left(E^{\prime}\right) \approx L / \tau\left(E^{\prime}\right)$, where $\tau\left(E^{\prime}\right)$ is the opacity of the EBL. The numerical constants $h$, and $k$ are the Planck constant, and Boltzmann constant respectively. A number of simplifying assumptions are implicit in equation 2.4. Firstly, we have assumed that the IGMF is coherent over the cooling length of the electrons, making the model valid for coherence lengths $\lambda_{B} \gtrsim 1 \mathrm{Mpc}$. We have neglected cosmological effects (except in the calculation of $\lambda\left(E^{\prime}\right)$ ) making the model valid for relatively small redshifts $z \lesssim 0.2$. We have only considered secondary photons (and no higher generations), and finally that each of the electrons and positrons produced in pair production interactions carries exactly $1 / 2$ of the energy of the primary photon. As discussed in [19], these are reasonable approximations that do not significantly affect the accuracy of the results.

For the injected spectrum, $f\left(E^{\prime}, \theta_{s}\right)$, we assume a boosted power law with index $\alpha$, and Lorentz factor $\left.\Gamma=1 / \sqrt{(} 1-\beta^{2}\right)$

$$
\begin{aligned}
f\left(E^{\prime}, \theta_{s}\right) & =f_{0}\left(1-\beta \cos \left(\theta_{s}\right)\right)^{(-\alpha-1)} E^{\prime-\alpha} e^{-E^{\prime} / E_{0}} \\
& +f_{0}\left(1+\beta \cos \left(\theta_{s}\right)\right)^{(-\alpha-1)} E^{\prime-\alpha} e^{-E^{\prime} / E_{0}} .
\end{aligned}
$$

Here, $E_{0}$ is the break energy, $\Gamma$ the bulk Lorentz factor, and $\alpha$ the injection spectral index. The second term in 2.5 is the contribution of the counter-jet which is non-negligible, especially in misaligned sources.

To model blazar spectra we limit $\theta_{c}$, requiring that it be smaller than the $68 \%$ containment radius of the Fermi point spread function (PSF) in the GeV range, and smaller than the Cherenkov 
telescope $\theta^{2}$ cut in the VHE range. For the Fermi PSF we use an analytical approximation, $\theta_{\mathrm{PSF}}=1.7^{\circ}\left(E_{\gamma, \mathrm{GeV}}\right)^{-0.74}\left[1+\left(E_{\gamma, \mathrm{GeV}} / 15\right)^{2}\right]^{0.37}$, valid in the range $30 \mathrm{MeV}$ to $300 \mathrm{GeV}$ [13]. The attenuated spectrum of the source is modelled as $f\left(E^{\prime}, \theta_{s}\right) \times \exp \left(-\tau\left(E^{\prime}\right)\right)$, where the EBL opacity is calculated using the model of [27]. To model a source with a limited activity cycle, we consider the time delay of the deflected emission following [19]

$$
c \Delta t=L^{\prime}+\sqrt{L^{2}+L^{\prime 2}-2 L L^{\prime} \cos \left(\theta_{d}-\theta_{c}-\theta_{v}\right)}-L .
$$

\section{Results}

We illustrate our method by considering the spectrum and expected halo emission of the blazar 1ES 1101+232, under different assumptions for the IGMF strength. The high-frequency peaked 1ES $1101+232$ is one of the 24 blazars in the stacking analysis of [28] that has resulted in a hint for halo emission at the $\sim 2.3 \sigma$ level, and in that sense, a potentially interesting candidate source for further pair halo studies. It is, however, possible to fit the spectrum of 1ES 1101+232 without requiring a non-zero magnetic field strength, as shown in [29]. Therefore, we use this source here for illustrative purposes primarily.

In figure 2 we show the expected spectrum of 1ES 1101+232 under different assumptions for the activity cycle of the gamma-ray source, and the intergalactic magnetic field strength. We have assumed the injected spectrum follows a power-law as in equation 2.5 with $\alpha=-1.5, E_{0}=10 \mathrm{TeV}$, and a bulk Lorentz factor $\Gamma=10$. As can be seen from the data-points in figure 2, a hard injected spectrum, and large maximum energy are needed at injection, in order to explain the observations of $1 \mathrm{ES} 1101+232$ at $\gtrsim 10 \mathrm{TeV}$ by HESS. The data-points are from observations made with HESS presented in [30], and the analysis of Fermi data therein. We show the spectrum of 1ES 1101+232 in two extreme limits, under the assumption that the gamma-ray source has been (a) active long enough to reach steady state $\sim 10^{6}$ years, or (b) only in the last $\sim$ decade, since its discovery by HESS.

Figure 3 shows the expected angular profile of 1ES 1101+232. The calculations presented here have been performed with ELMAG. The left panel shows the expected halo for different IGMF strengths, assuming the source has reached steady state, with stronger magnetic fields resulting in a more extended halo, as might be naively expected. The right panel shows the expected angular brightness profile of 1ES 1101+232 assuming different lengths of the gamma-ray activity of the source. A smaller source extension is expected if the source has been active for a shorter time, meaning that a transient, or short lived source, produces a narrower halo than a steady source at a given IGMF strength and energy range.

\section{Discussion}

We have presented a complementary approach to Monte Carlo, to model the expected gammaray emission of blazars after extragalactic propagation. The formalism we have presented, builds on earlier studies by $[19,18]$ and references therein, but goes beyond these earlier works in that the inverse Compton process is treated explicitly in the Thompson and Klein-Nishina regimes, and that our formalism generalises to sources of all orientations. This is an important novel feature, as we 

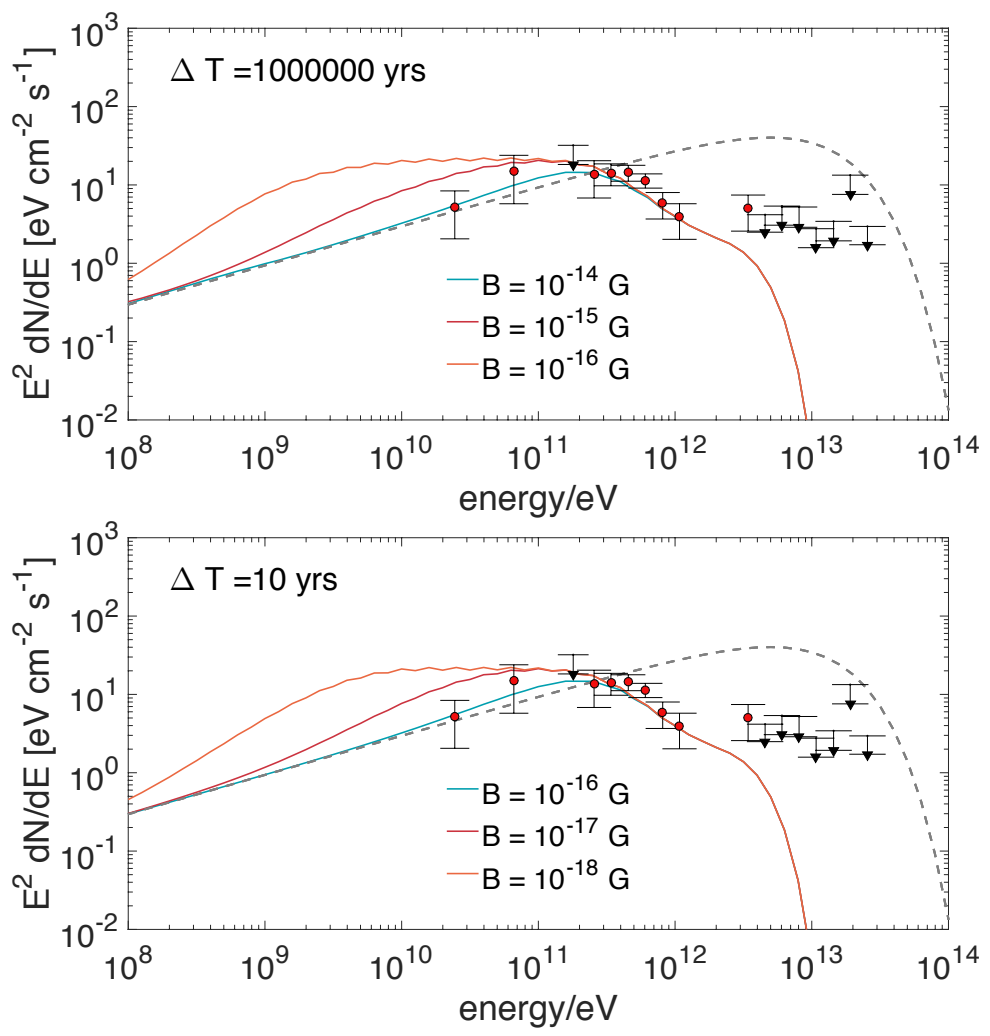

Figure 2: Spectrum of 1ES 1101+232 as observed with Fermi and HESS [30], and model predictions for different values of the average extragalactic magnetic field strength, $B$, for a source with an unlimited activity cycle (top), and a 10 year activity cycle (bottom).
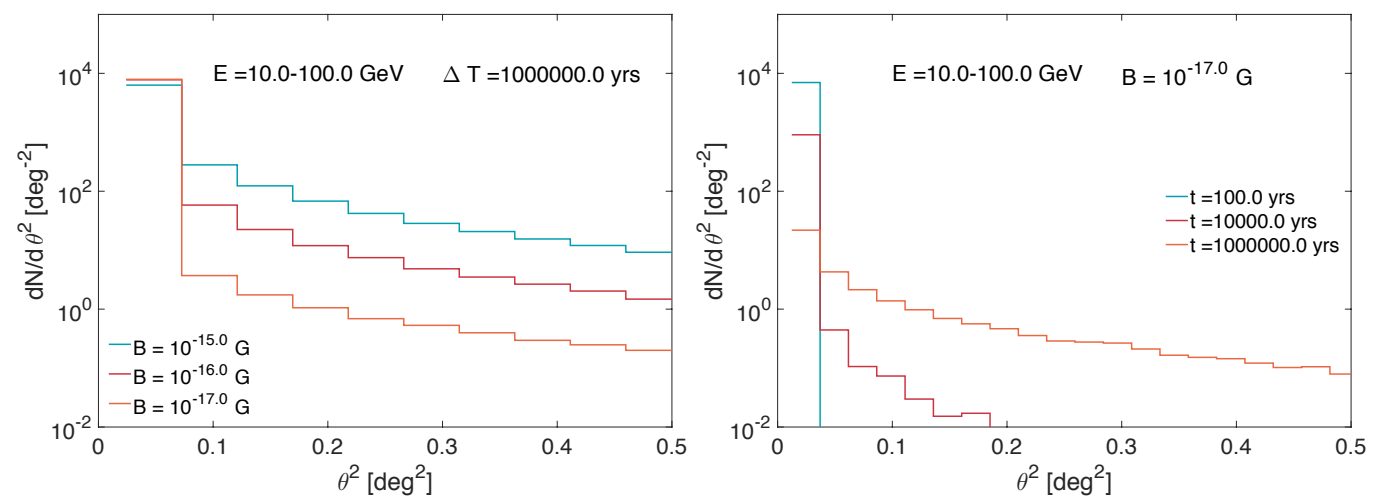

Figure 3: Left: Halo of 1ES 1101+232 assuming the gamma-ray source in 1ES 1101+232 has been active for $10^{6}$ years for different intergalactic magnetic field strengths. Right: Halo of 1ES 1101+232 for prompt $(<100 \mathrm{yrs})$, and delayed $\left(>100 \mathrm{yrs}\right.$ and $\left.<10^{4} \mathrm{yrs}\right)$, and $\left(>10^{4} \mathrm{yrs}\right.$ and $\left.<10^{6}\right)$ yrs emission. 
aim to harness information from observations of radio galaxies, which are much more numerous than blazars, in order to constrain IGMFs, as we will show in forthcoming work. Further, we have presented the dependence of the halo size on the delay time with respect to emission, demonstrating that halos produced by transient sources can reveal the IGMF strength, by their varying size as a function of time.

\section{References}

[1] K.-T. Kim, P. P. Kronberg and P. C. Tribble, Detection of excess rotation measure due to intracluster magnetic fields in clusters of galaxies, ApJ 379 (Sept., 1991) 80-88.

[2] D. Ryu, H. Kang and P. L. Biermann, Cosmic magnetic fields in large scale filaments and sheets, A\&A 335 (July, 1998) 19-25, [astro-ph/9803275].

[3] T. E. Clarke, P. P. Kronberg and H. Böhringer, A New Radio-X-Ray Probe of Galaxy Cluster Magnetic Fields, ApJl 547 (Feb., 2001) L111-L114, [astro-ph/ 0011281 ].

[4] C. L. Carilli and G. B. Taylor, Cluster Magnetic Fields, ARAA 40 (2002) 319-348, [astro-ph/0110655].

[5] J. P. Vallée, Observations of the Magnetic Fields Inside and Outside the Milky Way, Starting with Globules ( 1 parsec), Filaments, Clouds, Superbubbles, Spiral Arms, Galaxies, Superclusters, and Ending with the Cosmological Universe's Background Surface (at 8 Teraparsecs), Fundamentals of Cosmic Physics 19 (1997) 1-89.

[6] R. Beck, Galactic and Extragalactic Magnetic Fields, in AIP Conf Series (F. A. Aharonian et al., eds.), vol. 1085, p. 83, Dec., 2008. DOI.

[7] R. Durrer and A. Neronov, Cosmological magnetic fields: their generation, evolution and observation, 21 (June, 2013) 62, [1303.7121].

[8] P. P. Kronberg and M. Simard-Normandin, New evidence on the origin of rotation measures in extragalactic radio sources, 263 (Oct., 1976) 653-656.

[9] P. P. Kronberg and J. J. Perry, Absorption lines, Faraday rotation, and magnetic field estimates for QSO absorption-line clouds, ApJ 263 (Dec., 1982) 518-532.

[10] P. Blasi, S. Burles and A. V. Olinto, Cosmological Magnetic Field Limits in an Inhomogeneous Universe, ApJl 514 (Apr., 1999) L79-L82, [astro-ph/9812487].

[11] M. S. Pshirkov, P. G. Tinyakov and F. R. Urban, New limits on extragalactic magnetic fields from rotation measures, Phys. Rev. Lett. 116 (May, 2016) 191302.

[12] A. Neronov and I. Vovk, Evidence for Strong Extragalactic Magnetic Fields from Fermi Observations of TeV Blazars, Science 328 (Apr., 2010) 73-, [1 006.3504 ].

[13] A. M. Taylor, I. Vovk and A. Neronov, Extragalactic magnetic fields constraints from simultaneous GeV-TeV observations of blazars, 529 (May, 2011) A144, [1101.0932].

[14] M. Ahlers and J. Salvado, Cosmogenic gamma rays and the composition of cosmic rays, 84 (Oct., 2011) 085019, [1105.5113].

[15] I. Vovk, A. M. Taylor, D. Semikoz and A. Neronov, Fermi/LAT Observations of 1ES 0229+200: Implications for Extragalactic Magnetic Fields and Background Light, 747 (Mar., 2012) L14, [1112.2534]. 
[16] K. Murase, K. Takahashi, S. Inoue, K. Ichiki and S. Nagataki, Probing Intergalactic Magnetic Fields in the GLAST Era through Pair Echo Emission from TeV Blazars, 686 (Oct., 2008) L67-L70, [0806.2829].

[17] K. Dolag, M. Kachelriess, S. Ostapchenko and R. Tomàs, Lower Limit on the Strength and Filling Factor of Extragalactic Magnetic Fields, 727 (Jan., 2011) L4, [1009.1782].

[18] C. D. Dermer, M. Cavadini, S. Razzaque, J. D. Finke, J. Chiang and B. Lott, Time Delay of Cascade Radiation for TeV Blazars and the Measurement of the Intergalactic Magnetic Field, 733 (June, 2011) L21, [1011.6660].

[19] H. Huan, T. Weisgarber, T. Arlen and S. P. Wakely, A New Model for Gamma-Ray Cascades in Extragalactic Magnetic Fields, 735 (July, 2011) L28, [1106.1218].

[20] T. C. Arlen, V. V. Vassiliev, T. Weisgarber, S. P. Wakely and S. Yusef Shafi, Intergalactic Magnetic Fields and Gamma Ray Observations of Extreme TeV Blazars, ArXiv e-prints (Oct., 2012), [1210.2802].

[21] A. Neronov and D. Semikoz, Sensitivity of gamma-ray telescopes for detection of magnetic fields in intergalactic medium, Phys.Rev. D80 (2009) 123012, [0 910.1920 ].

[22] A. Elyiv, A. Neronov and D. V. Semikoz, Gamma-ray induced cascades and magnetic fields in the intergalactic medium, 80 (July, 2009) 023010, [0 903.3649 ].

[23] K. Dolag, M. Kachelrieß, S. Ostapchenko and R. Tomàs, Blazar Halos as Probe for Extragalactic Magnetic Fields and Maximal Acceleration Energy, 703 (Sept., 2009) 1078-1085, [0903. 2842].

[24] M. Kachelriess, S. Ostapchenko and R. Tomas, ELMAG: A Monte Carlo simulation of electromagnetic cascades on the extragalactic background light and in magnetic fields, Comput.Phys.Commun. 183 (2012) 1036-1043, [1106.5508].

[25] K. Ichiki, S. Inoue and K. Takahashi, Probing the Nature of the Weakest Intergalactic Magnetic Fields with the High-Energy Emission of Gamma-Ray Bursts, 682 (July, 2008) 127-134, [0711.1589].

[26] G. R. Blumenthal and R. J. Gould, Bremsstrahlung, Synchrotron Radiation, and Compton Scattering of High-Energy Electrons Traversing Dilute Gases, Reviews of Modern Physics 42 (1970) 237-271.

[27] A. Franceschini, G. Rodighiero and M. Vaccari, The extragalactic optical-infrared background radiations, their time evolution and the cosmic photon-photon opacity, Astron.Astrophys. 487 (2008) 837, [0805.1841].

[28] W. Chen, J. H. Buckley and F. Ferrer, Search for GeV gamma-Ray Pair Halos Around Low Redshift Blazars, Phys. Rev. Lett. 115 (2015) 211103, [1410 . 7717].

[29] A. Neronov and I. Vovk, Evidence for strong extragalactic magnetic fields from Fermi observations of TeV blazars, Science 328 (2010) 73-75, [1006.3504].

[30] H.E.S.S. collaboration, A. Abramowski et al., Search for Extended gamma-ray Emission around AGN with H.E.S.S. and Fermi-LAT, Astron. Astrophys. 562 (2014) A145, [1401.2915]. 